\title{
Prediction of equivalent static loads act on a micro satellite via modal analysis
}

\author{
M. Safarabadi ${ }^{a^{*}}$ and S. Bazargan ${ }^{\mathrm{b}}$
}

${ }^{a}$ School of Mechanical Engineering, College of Engineering, University of Tehran, Tehran, Iran ${ }^{b}$ Aerospace Engineering Faculty, KNT University of Technology, Tehran, Iran

\begin{tabular}{|c|c|}
\hline ARTICLE INFO & A B S T RACT \\
\hline $\begin{array}{l}\text { Article history: } \\
\text { Received October 6, } 2014 \\
\text { Accepted } 2 \text { February } 2015 \\
\text { Available online } \\
\text { 2 February } 2015 \\
\text { Keywords: } \\
\text { Quasi-static load } \\
\text { Random vibration } \\
\text { Low frequency load } \\
\text { Equivalent static load } \\
\text { Shock Phenomenon } \\
\text { Modal effective mass }\end{array}$ & $\begin{array}{l}\text { In this research, the influences of modal properties of a micro cubic satellite on equivalent } \\
\text { static loads (due to combination of quasi-static and dynamic loads during launch time) have } \\
\text { been studied. The study shows that the magnitude of equivalent static loads can be affected by } \\
\text { satellite effective modes and effective mass distribution along natural frequencies. Besides, } \\
\text { when to distinct launchers with different dynamic environments candidate for launch, } \\
\text { analytical results illustrate that sometimes the equivalent static combined load value can be } \\
\text { higher for the launcher with smaller quasi-static loads. This phenomenon is due to effective } \\
\text { modal mass distribution of the satellite. Consequently, a higher combined load may be } \\
\text { occurred during launch for smaller quasi-static conditions. Furthermore, in separation phase of } \\
\text { satellite from the launcher, the satellite modal parameters influence the magnitude of applied } \\
\text { shock load in both axial (launch) and lateral directions, importantly. Thus the present study } \\
\text { yields reliable input values for separation shock test which considers dynamic properties of the } \\
\text { satellite in comparison with some standards and launcher manuals, suggest a rough estimation } \\
\text { of shock load, neglecting the satellite structure dynamic behavior. }\end{array}$ \\
\hline
\end{tabular}

\section{Introduction}

The satellite structure must withstand the static and dynamic loads during launch and injection into its final orbit. The launch process conditions may induce nearly constant acceleration to the satellite. Usually these acceleration values introduce as multiplies of gravitational acceleration in three main direction (one along the launch direction, and two in lateral directions). These loads are defined as quasi-static loads (Larson \& Wertz, 1999). On the other hand, other dynamic loads exist during launch which can be added to the above mentioned quasi-static loads. These dynamic loads include random vibration load, low frequency sine load and acoustic vibrations. These loads are due to launcher engine and its related parts and components. During lift-off, the main cause of random vibrations is the acoustic loads due to engine noise, the aerodynamic forces due to supersonic speed achieved by the launcher, is

* Corresponding author

E-mail addresses: msafarabadi@ut.ac.ir (M. Safarabadi) 
another source of random vibration during launch. These dynamic loads are added to the quasi-static loads and consequently, several combined load conditions can be occurred (Safarin, 1995).

Another source of applied dynamic load is due to shock phenomenon, resulting from separation of satellite from its launcher. This load must be introduced as an equivalent static load too (Himelblau et al., 2001). Hence, in order to perform a stress analysis for the satellite structure, the integration of quasistatic and dynamic loads is required. Because of various nature of the two mentioned loads (static and dynamic) and an equivalent static load is considered during launch in order to combination of them.

Consequently, in this paper, based on the modal analysis results, these equivalent static loads are predicted and the effects of satellite effective modes distribution on these loads is investigated. For this purpose, a finite element model of the satellite is build up and a modal analysis is performed using MSC Nastran/MSC Patran software. The consequent effective modes and the corresponding effective mass is employed for prediction of the equivalent static loads due to random vibrations, low frequency sine vibration and shock loads, separately. The results show that the effective modes distribution influences the equivalent static loads. In other words, the effective modal mass plays an important role in dynamic loads act on the satellite. So, the total applied loads to the satellite are dependent upon its dynamic characteristics. Thus, in order to minimize loads act on the satellite structure, an optimum design for satellite structure could be achieved which leads to an optimum modal effective modes distribution. Consequently, non-destructive stresses and an acceptable margin of safety is obtainable during launch.

\section{Loads applied on the satellite during launch}

A satellite structure provides the necessary mechanical support for the whole satellite subsystems. The structure must withstand the static and dynamic loads from the launcher during the satellite launch and injection in to its final orbit. The satellite base plate is attached to the launcher through an interface. In about ten minutes during which the launcher transfers the satellite to its final orbit, the satellite structure is acted upon by static and dynamic loads. These loads are considered in the design of the satellite structure. The propulsive thrust of the launcher produces an almost constant propulsive force along the launcher axis. Acceleration increases slightly with the decrease in the launcher fuel mass, and ceases completely on complete consumption of the fuel. Satellite launchers are usually of multistage type. Therefore, the above process may induce nearly constant acceleration to the satellite with different intensities. Satellite manufacturers usually present the acceleration values as multiples of gravitational (quasi-static loads) acceleration in three main directions (one along the launch direction, and two in direction perpendicular to the launch direction). These acceleration values basically demonstrate the inertial phenomenon, which is applied to all the satellite components in the form of inertial forces (Larson \& Wertz, 1999). Table 1 introduces the quasi-static loads based on the information reported in Cosmos launch systems (1999).

Table 1. Quasi-static load magnitudes for Cosmos launch vehicle as a factor of gravitational acceleration

\begin{tabular}{ccc}
\hline Launch Vehicle Type & Longitudinal (launch) Direction & Lateral Directional \\
\hline Cosmos & $6.8 \mathrm{~g}$ & $1.6 \mathrm{~g}$ \\
\hline
\end{tabular}

In addition to this effect, gravitational acceleration graphs based on frequency show the transient effect of this phenomenon at low frequencies (between 5 to $100 \mathrm{~Hz}$ ). Table 2 show the level of these sinusoidal vibrations for Cosmos launcher (Cosmos Launch System, 1999).

Table 2. Low frequency sinusoidal loads for Cosmos launcher as a factor of gravitational acceleration in both longitudinal and lateral directions

\begin{tabular}{ccccc}
\hline Frequency band [Hz] & $5-20$ & $20-40$ & $40-65$ & $65-100$ \\
\hline Amplitude [g] & 0.5 & 0.7 & 1.0 & 1.7 \\
\hline
\end{tabular}


Another source of applied load on the satellite structure during the launch period is the random vibrations due to launcher engine and its related parts and components. During lift off, the main cause of these random vibrations is the acoustic loads due to engine noise. Beside this factor, the random vibrations are caused by aerodynamic forces due to supersonic speed achieved by the launcher. The combination of these vibrations is transmitted to the satellite through the rocket structure (Larson \& Wertz, 1999). At the same time, satellite structural elements, with large area to mass ratios (e.g. solar panels) are directly and severely affected by the acoustic phenomenon. Loads resulting from random phenomenon cannot be expressed as multiple of gravitational acceleration. The three variables which help describe the random vibrations are: type of distribution, frequency content, and magnitude of these vibrations (Wijker, 2003).

In general, it is assumed that the random phenomenon has a Gaussian distribution, and in this manner the type of intensity distribution in time is defined. For expression of frequency content, the power spectral density (PSD) is used (for this specific application, the term acceleration is a better choice). An acceleration spectral density (ASD) at frequency $\mathrm{f}$, is the mean-square acceleration within a selected frequency band (whose center is $\mathrm{f}$ ) divided by the bandwidth. Commonly, PSD is expressed in units of $g^{2} / \mathrm{Hz}$, where $\mathrm{g}$ is the acceleration of Earth's gravity at sea level (Safarin, 1995). To show the PSD, it is plotted on log-log paper, typically covering the frequency range of 20 to $2000 \mathrm{~Hz}$ which has been found to adequately describe random vibration for structures and electronic components. The random vibration loads of Cosmos launcher (Cosmos Launch System, 1999) is presented in Table 3.

Table 3. Spectral density of random vibration acceleration for Cosmos launcher.

\begin{tabular}{cc}
\hline Frequency $[\mathbf{H z}]$ & PSD [g2/Hz] \\
\hline $20-50$ & $0.004-0.01$ \\
$50-125$ & $0.01-0.11$ \\
$125-135$ & 0.11 \\
$135-145$ & $0.11-0.08$ \\
$145-200$ & 0.08 \\
$200-2000$ & $0.08-0.016$ \\
\hline
\end{tabular}

The third source of applied load to the structure is due to the shock phenomenon, resulting from separation of satellite from its launcher. To produce the suitable initial velocity in the satellite, it is necessary to apply a big acceleration in a very short period. This may be achieved using explosive mechanisms. Other phenomena such as separation of antenna and deployment of solar panels can also cause shock. However, normally almost all of the shock phenomenon is caused by separation of satellite from its launcher. Shock environment is described by a shock spectrum (Sarafin and Larson 1995) Table 4 presents the maximal values of the shock loads for Cosmos launch vehicle (Cosmos Launch System, 1999). The values include an amplification factor of $\mathrm{Q}=10$.

Table 4. The maximal values of the shock loads acceleration for Cosmos launcher

\begin{tabular}{ccc}
\hline Frequency band [Hz] & $100-400$ & $400-10000$ \\
\hline Amplitude [g] & $10-150$ & $150-400$ \\
\hline
\end{tabular}

The main loads due to launch conditions are placed in the three groups given above. The satellite structure due to the above conditions will bear reactive inertial forces due to the transmitted accelerations from the satellite base plate. The calculated stresses during the worst loading conditions are compared with the yield point and tensile strength to determine the safety factors. There is an acoustic environment induced by stage one engine operation and boundary layer noise. These loads are in this investigation ignored in comparison with the value of other mentioned loads and the small satellite dimensions. 
In the proto-qualification test phase and before launch also some loads related to mention loads act on the satellite. One of them is sinusoidal vibrations loads for the proto-qualification test which is equal with sinusoidal vibrations loads declared by launch vehicle manufacturers. Another test load is random vibrations loads for the proto-qualification test which its level is $+3 \mathrm{db}$ upper than random vibrations loads declared by launch vehicle manufacturers as is mentioned in the launch vehicle manuals according to standard ECSS-E-10-03A (Noordwijk, 2002).

\section{Calculation of equivalent static loads}

As mentioned earlier, during launch time, the quasi-static loads are combined with random vibrations and low frequency loads. So, in order to perform strength analysis for satellite structure and its subsystems, it is necessary to predict the equivalent static loads of these combined load conditions.

\subsection{Quasi-static load and random vibrations combination}

In the case of base excitation phenomenon of a system with one degree of freedom, consisting of spring and mass, with random vibration of constant amplitude of white noise type with constant frequency range of zero to infinity, the loads due to random vibrations can be expressed on basis of gravitational acceleration (Miles, 2012).

In recent years, a method has been developed for extension of this relation (equation) to include systems of $n$ degrees of freedom. It can be shown that using decoupled equations in a particular coordinate system, for base excitation phenomenon of a system with $n$ degrees of freedom, the dynamic characteristics of the system can be expressed on basis of normal modals and effective masses (Plesseria et al., 2000; Emami \& Safarabadi, 2007; Bohlouri et al., 2014). The normal modes and effective masses define specific directions in which the acceleration is independent and is related to the frequency and mode shape of that frequency. On the other hand, the absorbed energy for movement in that specific direction is linearly related to effective mass that is defined for that particular mode. The total effective masses are equivalent to the total mass of structure, and therefore, amount of effective mass for each mode expresses the specific share of that mode in the total vibrations of the system (Plesseria et al., 2000; Emami \& Safarabadi, 2007; Safarabadi \& Emami, 2008; Safarabadi \& Emami, 2009).

Under such conditions, the Miles equation for systems with $\mathrm{n}$ degrees of freedom can be expressed as following:

$$
\begin{aligned}
& n_{i}=\sqrt{\pi / 2 \times f_{i} \times Q \times W_{i}} \\
& p_{i}=n_{i} \times g \times m_{e f f, i} \\
& R V L=3 \times \frac{\sqrt{\sum P_{i}^{2}}}{\sum m_{e f f, i} \times g}
\end{aligned}
$$

The equivalent load factor as a multiple of gravitational acceleration is calculated using Eq. (1). In the above equations, $f_{i}$ and $W_{i}$ are natural frequency and PSD value at $i^{\text {th }}$ mode respectively. $Q$ is the amplification factor. Value of $p_{i}$ for each of the selected modes is calculated using Eq. (2). In this

equation, $m_{\text {eff }, i}$ is the effective mass obtained at $i^{\text {th }}$ mode and gravitational acceleration g. Eq. (3) yields the random vibration load factor $(R V L)$ which considers a factor equal to 3 according to statistical results and uncertainties (Plesseria et al., 2000; Safarabadi \& Emami, 2009).

Table 5 presents the load cases that may be occurred due to combination of quasi-static loads and random vibrations during launch. In this table, $Q S L$ and $R V L$ introduces quasi-static and random vibration load factors respectively. 
Table 5. Combination of quasi-static and random vibration loads at launch time.

\section{LOAD CASE $\quad$ Lateral Direction (Y) $\quad$ Lateral Direction (Z) $\quad$ Axial Direction (X)}

\begin{tabular}{cccc}
\hline 1 to 8 & $\pm \sqrt{Q S L_{A X}^{2}+R V L_{A X}^{2}}$ & $\pm Q S L_{L A T}$ & $\pm Q S L_{L A T}$ \\
\hline 9 to 16 & $\pm Q S L_{A X}$ & $\pm \sqrt{Q S L_{L A T}^{2}+R V L_{L A T-Y}^{2}}$ & $\pm Q S L_{L A T}$ \\
\hline 17 to 24 & $\pm Q S L_{A X}$ & $\pm Q S L_{L A T}$ & $\pm \sqrt{Q S L_{L A T}^{2}+R V L_{L A T-Z}^{2}}$ \\
\hline
\end{tabular}

\subsection{Quasi-static load and low frequency sinusoidal vibrations combination}

Harmonic sinusoidal vibrations are applied to the satellite at low frequencies (between 5-100 Hz). These loads can be introduces as a factor of gravitational acceleration $(\mathrm{g})$ based on below equation:

$$
S L_{S I N E}=g_{\text {INPUT }} \times Q \times S F
$$

where $S L_{S I N E}$ is the equivalent static load due sinusoidal vibrations. $g_{\text {INPUT }}$ is the magnitude of low frequency load (which is presented in launcher manual) at three first effective frequencies of the satellite in axial and lateral directions. $Q$ and $S F$ are amplification factor and load factor of safety respectively. As mentioned before, the harmonic sinusoidal low frequency vibrations integrate with quasi-static loads during launch. Table 6 presents the equivalent static loads along three directions (axial and lateral) induced by this combination (Emami et al., 2008).

Table 6. Combination of quasi-static and low frequency vibration loads at launch time.

\begin{tabular}{cccc}
\hline LOAD CASE & Lateral Direction (Y) & Lateral Direction (Z) & Axial Direction (X) \\
\hline $25-32$ & $\pm\left(S L_{S I N E}+Q S L_{L A T}\right)$ & $\pm Q S L_{L A T}$ & $\pm Q S L_{A X}$ \\
\hline $33-40$ & $\pm Q S L_{L A T}$ & $\pm\left(S L_{S I N E}+Q S L_{L A T}\right)$ & $\pm Q S L_{A X}$ \\
\hline $41-48$ & $\pm Q S L_{L A T}$ & $\pm Q S L_{L A T}$ & $\pm\left(S L_{S I N E}+Q S L_{A X}\right)$ \\
\hline
\end{tabular}

\subsection{Calculation of equivalent static loads due to separation (shock phenomenon)}

Of the three groups of loads acting on the structure, the third group, the forces resulting from the shock, act on the structure when different modules in the launcher propulsion system have completed their functions. The first two groups can appear in combination. The resultant loads due to the shock phenomenon are act on the structure, independently. Intensity of the equivalent shock force on the structure in any direction equals the maximum force obtained for different modes in that particular direction, and is obtained using the following relation (Emami et al., 2008):

$$
g_{\text {Equvalent Shock }}=S_{i} \times \frac{m_{e f f, i}}{M_{\text {Sat }}}
$$

In Eq. (5) $m_{e f f, i}$ and $M_{s a t}$ are the effective mass obtained at $i^{\text {th }}$ mode and total mass of the satellite, respectively. $S_{i}$ is the magnitude of shock load (according to the launcher manual) at effective modes. Table 7 introduces the load states due to separation (Emami et al., 2008).

Table 7. The equivalent shock loads in three main directions.

\section{$\begin{array}{llll}\text { LOAD CASE } & \text { Lateral Direction (Y) } & \text { Lateral Direction (Z) } & \text { Axial Direction (X) }\end{array}$}

\begin{tabular}{cccc}
\hline $49-50$ & $\pm\left(g_{\text {Equvalent Shock }}\right)_{Y}$ & - & - \\
$51-52$ & - & $\pm\left(g_{\text {Equvalent Shock }}\right)_{Z}$ & - \\
$53-54$ & - & - & $\pm\left(g_{\text {Equvalent Shock }}\right)_{X}$ \\
\hline
\end{tabular}




\section{The finite element model of the satellite}

In this research work, a cubic satellite has been modeled in MSc/Patran software. Vibration sensitive components such as electronic boards are packed in an electronic box, in contact with the base plate. In this location, the electronic boards withstand the minimum vibrations transmitted from the launcher. The satellite structure dimensions are: $420 * 500 * 500\left(\mathrm{~mm}^{3}\right)$, and the total structure mass including all the internal components is $42 \mathrm{~kg}$. Modeling of components has been done using solid elements. Modeling of joints has been done using merge nodes in location of joints. Mechanisms also have been modeled by MPCs. Small non-structural elements (such as sun sensors, etc.) have been modeled using concentrated mass element. For modeling of bolts, rivets, thermal control hardware, and electrical harness, non-structural mass elements have been used. Fig. 1 shows the finite element model of the satellite. As it is seen, $\mathrm{X}$ direction is correspond to axial (launch) direction, while $\mathrm{Y}$ and $\mathrm{Z}$ denotes lateral directions. Type and number of elements used in this FE model are presented in Table 8.
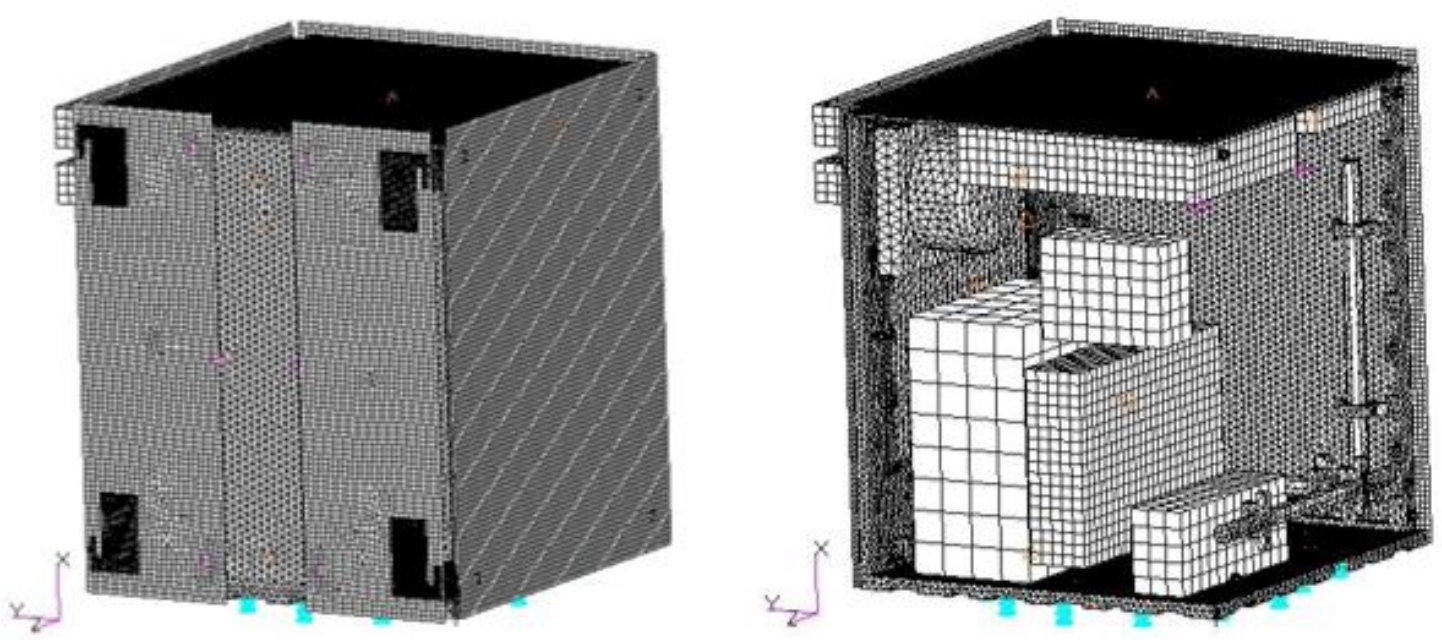

Fig. 1. The satellite FE model

Table 8. The FE model specifications

\begin{tabular}{cc}
\hline Element Type & Number \\
\hline GRID POINTS & 102957 \\
ELEMENTS & 250572 \\
CBAR & 160 \\
CHEXA & 5274 \\
CONM2 & 10 \\
CQUAD4 & 28795 \\
CTRIA3 & 54 \\
CTETRA & 216267 \\
RBE2 & 12 \\
\hline
\end{tabular}

\section{Results and discussions}

In this section the results from the finite element analysis are presented. Based on the modal analysis, the modal effective modes and their corresponding masses is obtained. Then by employing the information of Cosmos user manual (which is presented in section 3 of this study) the equivalent static load cases are presented. Finally, an investigation is performed in order to compare the loads applied to the satellite by to various launch environments. 
Table 9 presents the effective modes, their direction and related effective masses (more than 2\%), that was obtained from modal analyses. As it is seen, the main three modes are the 1st, 2nd and 6th modes which include two bending modes and one axial mode with the effective mass more than $50 \%$. Besides, most of the effective modes are happened in axial (launch) direction. The cumulative effective mass is close to satellite total mass in each direction.

Table 9. Effective modes, direction and related effective masses

\begin{tabular}{ccccc}
\hline Mode Number & $\begin{array}{c}\text { Frequency } \\
(\mathrm{Hz})\end{array}$ & \multicolumn{3}{c}{ Effective Mass (\%) } \\
\cline { 3 - 5 } & 63.11 & Lateral direction $(\mathrm{Y})$ & Lateral direction $(\mathrm{Z})$ & Axial (Launch) direction $(\mathrm{X})$ \\
\hline 1 & $\mathbf{5 0 . 4 2 \%}$ & $<1 \%$ & $<1 \%$ \\
2 & 102.47 & $<1 \%$ & $\mathbf{5 6 . 0 9 \%}$ & $<1 \%$ \\
4 & 171.49 & $\mathbf{2 . 3 2 \%}$ & $<1 \%$ & $\mathbf{3 . 1 7 \%}$ \\
5 & 176.74 & $<1 \%$ & $<1 \%$ & $\mathbf{2 . 5 1 \%}$ \\
6 & 189.92 & $<1 \%$ & $<1 \%$ & $\mathbf{5 0 . 5 5 \%}$ \\
17 & 287.93 & $<1 \%$ & $\mathbf{3 . 7 4 \%}$ & $\mathbf{1 1 . 5 7 \%}$ \\
20 & 339.70 & $\mathbf{2 . 7 \%}$ & $<1 \%$ & $<1 \%$ \\
28 & 407.90 & $<1 \%$ & $\mathbf{2 . 1 4 \%}$ & $<1 \%$ \\
36 & 535.53 & $\mathbf{6 . 1 8 \%}$ & $<1 \%$ & $\mathbf{2 . 0 4 \%}$ \\
57 & 771.31 & $<1 \%$ & $\mathbf{2 . 9 2 \%}$ & $\mathbf{4 . 7 \%}$ \\
77 & 920.91 & $<1 \%$ & $<1 \%$ & $\mathbf{3 . 3 1 \%}$ \\
\hline
\end{tabular}

Tables 10 and 11 show the equivalent static load factor (as a factor of gravitational acceleration $\mathrm{g}$ ) due to combination of quasi-static load with random vibrations and low frequency sinusoidal loads respectively. As shown in Table 10 a maximum value of combined axial acceleration equal to $33.2 \mathrm{~g}$ occurs, while the quasi static magnitude of the Cosmos launcher is $6.8 \mathrm{~g}$ along launch direction. Besides, the value of lateral equivalent loads are $4.9 \mathrm{~g}$, which is comparable with its corresponding amplitude (equal to $1.6 \mathrm{~g}$ ) reported in Cosmos user manual. Similar results are obtained when quasi-static loads are combined with low frequency vibrations (load cases 25-48) as shown is Table 11. Consequently, random and low frequency vibrations have very important role in the magnitude of loads which is applied to the satellite during launch. The quasi-static loads are very small in comparison with real loads.

Table 10. The equivalent static load due to combination of quasi-static and random vibration loads as a factor of gravitational acceleration $\mathrm{g}$.

\begin{tabular}{cccc}
\hline LOAD CASE & Lateral Direction $(\mathbf{Y})$ & Lateral Direction $(\mathbf{Z})$ & Axial Direction $(\mathbf{X})$ \\
\hline $1-8$ & \pm 4.9 & \pm 4.9 & \pm 33.2 \\
$9-16$ & \pm 4.9 & \pm 27.4 & \pm 9.3 \\
$17-24$ & \pm 13.9 & \pm 4.9 & \pm 9.3 \\
\hline
\end{tabular}

Table 11. The equivalent static load due to combination of quasi-static and low frequency vibrations as a factor of gravitational acceleration $\mathrm{g}$.

\begin{tabular}{cccc}
\hline LOAD CASE & Lateral Direction (Y) & Lateral Direction (Z) & Axial Direction (X) \\
\hline $25-32$ & \pm 4.9 & \pm 4.9 & \pm 31.4 \\
$33-40$ & \pm 4.9 & \pm 27 & \pm 9.3 \\
$41-48$ & \pm 27 & \pm 4.9 & \pm 9.3 \\
\hline
\end{tabular}

Table 12 presents the equivalent shock load occurred when the satellite separates from its launcher in final orbit. As it is seen, a considerable shock load equal to $17.03 \mathrm{~g}$ is obtained in axial (launch) direction. This maximum value depends on effective frequency and its corresponding effective mass. Consequently, the effective mass distribution play an important role in applied shock load to the satellite structure. Table 13 summarizes the calculations given in Table 12. 
Table 12. Equivalent static applied loads in separation (shock phenomenon) along axial and lateral directions.

\begin{tabular}{|c|c|c|c|c|c|c|c|c|c|c|c|}
\hline \multicolumn{4}{|c|}{ Lateral Direction (Y) } & \multicolumn{4}{|c|}{ Lateral Direction $(\mathbf{Z})$} & \multicolumn{4}{|c|}{ Axial Direction (X) } \\
\hline $\begin{array}{c}\text { Frequency } \\
(\mathrm{Hz})\end{array}$ & $S_{i}(\mathrm{~g})$ & $\frac{m_{e f f, i}}{M_{\text {Sat }}}$ & $g_{\text {Shock }}$ & $\begin{array}{c}\text { Frequency } \\
(\mathrm{Hz})\end{array}$ & $S_{i}(\mathrm{~g})$ & $\frac{m_{e f f, i}}{M_{\text {Sat }}}$ & $g_{\text {Shock }}$ & $\begin{array}{c}\text { Frequency } \\
(\mathrm{Hz})\end{array}$ & $S_{i}(\mathrm{~g})$ & $\frac{m_{e f f, i}}{M_{S a t}}$ & $g_{\text {Shock }}$ \\
\hline 63.11 & 0 & $50.42 \%$ & 0 & 102.47 & 12.5 & $56.09 \%$ & 7.01 & 171.49 & 64.50 & $3.17 \%$ & 2.05 \\
\hline 171.49 & 64.54 & $2.32 \%$ & 1.497 & 287.93 & 117.38 & $3.74 \%$ & 4.39 & 176.74 & 67.62 & $2.51 \%$ & 1.70 \\
\hline 339.7 & 133.6 & $2.70 \%$ & 3.607 & 407.90 & 151.47 & $2.14 \%$ & 3.24 & 186.92 & 73.25 & $50.55 \%$ & 17.03 \\
\hline 535.53 & 172.6 & $6.18 \%$ & 10.67 & 771.31 & 200.95 & $2.92 \%$ & 5.87 & 287.93 & 117.38 & $11.57 \%$ & 13.58 \\
\hline & & & & & & & & 535.53 & 172.61 & $2.04 \%$ & 3.52 \\
\hline & & & & & & & & 771.31 & 200.95 & $4.7 \%$ & 9.45 \\
\hline & & & & & & & & 920.91 & 214.71 & $3.31 \%$ & 7.11 \\
\hline
\end{tabular}

Table 13. Summarized shock loads form Table 12

LOAD CASE $\quad$ Lateral Direction (Y) $\quad$ Lateral Direction (Z) $\quad$ Axial Direction (X)

\begin{tabular}{cccc}
\hline $49-50$ & $\pm 10.67 g$ & - & - \\
$51-52$ & - & $\pm 7.01 g$ & - \\
$53-54$ & - & - & $\pm 17.03 g$ \\
\hline
\end{tabular}

Many satellite designers consider only the quasi static loads in order to compare two different launch vehicles. As it is seen, during launch these quasi-static loads integrate with dynamic loads (random and low frequency vibrations). Thus, several load conditions are obtained with very higher load magnitudes. Consequently, comparison of two different launchers cannot be acceptable when only the quasi-static loads are compared. Therefore, as a complementary to this study, another launch vehicle (Dnepr launcher) is considered (Dnepr Launch System, 2001). Table 14 shows the quasi-static load values of Cosmos and Dnepr launch vehicles.

Table14. Quasi-static load magnitudes for Cosmos and Dnepr launch vehicles

\begin{tabular}{ccc}
\hline Launch Vehicle Type & Longitudinal (launch) Direction & Lateral Directional \\
\hline Cosmos & $6.8 \mathrm{~g}$ & $1.6 \mathrm{~g}$ \\
Dnepr & $7.5 \mathrm{~g}$ & $2.1 \mathrm{~g}$ \\
\hline
\end{tabular}

Table 15 compares the equivalent static loads for cosmos and Dnepr launcher. As it is seen from this Table, the equivalent static loads for Cosmos launcher is higher than their corresponding values for Dnepr in axial and lateral directions, while the quasi-static load of Cosmos is smaller than its magnitude for Dnepr launch vehicle. This is strange phenomenon is due to the distribution of satellite effective modes and different in dynamic load distributions of the two launch vehicles along frequencies.

Table 15. Comparison of applied combined loads for the two launch vehicles.

\begin{tabular}{ccccccc}
\hline \multirow{2}{*}{ LOAD CASE } & \multicolumn{2}{c}{ Lateral Direction (Y) } & \multicolumn{2}{c}{ Lateral Direction (Z) } & \multicolumn{2}{c}{ Axial Direction (X) } \\
\cline { 2 - 6 } & Cosmos & Dnepr & Cosmos & Dnepr & Cosmos & Dnepr \\
\hline $1-8$ & \pm 4.9 & \pm 2.10 & \pm 4.9 & \pm 2.10 & \pm 33.2 & \pm 25 \\
$9-16$ & \pm 4.9 & \pm 2.10 & \pm 27.4 & \pm 23.1 & \pm 9.3 & \pm 7.5 \\
$17-24$ & \pm 13.9 & \pm 14.1 & \pm 4.9 & \pm 2.10 & \pm 9.3 & \pm 7.5 \\
$25-32$ & \pm 4.9 & \pm 2.10 & \pm 4.9 & \pm 2.04 & \pm 31.4 & \pm 29.6 \\
$33-40$ & \pm 4.9 & \pm 2.10 & \pm 27 & \pm 24.5 & \pm 9.3 & \pm 7.5 \\
$41-48$ & \pm 27 & \pm 24.5 & \pm 4.9 & \pm 2.10 & \pm 9.3 & \pm 7.5 \\
$49-50$ & \pm 10.67 & \pm 10.25 & - & - & - & - \\
$51-52$ & - & - & \pm 7.01 & \pm 8.93 & - & - \\
$53-54$ & - & - & - & - & \pm 17.03 & \pm 14.37 \\
\hline
\end{tabular}




\section{Conclusions}

In this paper, the equivalent static loads due to combination of quasi-static and dynamic loads during launch have been predicted based on modal analysis results. First, applied loads to the satellite have been described. Cosmos launcher is considered for this study and its quasi-static and dynamic loads are introduced according to its manual. In the next section, the philosophy of loads combination is presented. Finally, a finite element model of the satellite is made and a modal analysis has been performed.

Based on the mentioned procedure and by incorporating the modal analysis results and loads of Cosmos launcher, the equivalent static loads during launch are obtained. The results illustrates important role of random and low frequency vibrations which are added to the quasi-static loads during launch. The magnitude of equivalent static loads (which is due to combination of quasi-static load with random, sinusoidal vibration and shock loads) are mainly dependent upon satellite dynamic behavior. Besides, when two various launch vehicles are considered, the equivalent static load could be higher for the launcher with lower quasi static load. Consequently, in order to perform strength analysis for the satellite structure, equivalent combined loads must be considered, instead of quasi static loads only. Neglecting this reality leads to uncertain design of the satellite and thus the mechanical failure is possible. In this way, the satellite modal properties have important effect on the combined load magnitudes. The present research can be employed for design optimization of satellite structure in order to load minimization during launch. This is possible by an appropriate effective mass distribution via changing satellite geometry, optimum positioning of its subsystems, structural stiffening and etc.

\section{Acknowledgment}

The authors would like to thank the anonymous referees for constructive comments on earlier version of this paper.

\section{References}

Bohlouri, V., Kosari, A., \& Aliha, M.R.M. (2014). Systematic design of an atmospheric data acquisition flying vehicle telemetry system. Engineering Solid Mechanics, 2(4), 265-276.

Cosmos Launch System. (1999). Payload User's Manual 2.1.

Dnepr Launch System. (2001). Payload SLS User's Guide.

Emami, H., \& Safarabadi, M. (2007). Investigation the effect of rigidity and geometry of a cubic satellite lateral plates on dynamic behavior of the structure during launch. 6th International Aerospace Conference, KNTU University, in Persian.

Emami, H., Farhani, F., Safarabadi, M. (2008). Influence of modal effective mass distribution on the static and dynamic behavior of a satellite under base excitations. Material Research India Journal.

Himelblau, H., Kern, D. L., Manning, J. E., Piersol, A. G., \& Rubin, S. (2001). Dynamic environmental criteria. NASA Technical Handbook, 7005.

Larson, W. J., \& Wertz, J. R. (1999). Space mission analysis and design (No. DOE/NE/32145--T1). Microcosm, Inc., Torrance, CA (US).

Miles, J. W. (2012). On structural fatigue under random loading. Journal of the Aeronautical Sciences (Institute of the Aeronautical Sciences), 21(11).

Noordwijk. (2002). ECSS-E-10-03A, Space engineering: testing. the Netherlands.

Plesseria, J. Y., Rochus, P., \& Defise, J. M. Effective modal masses.

Safarabadi, M., \& Emami, H. (2008). The Influence of effective mass distribution and local behavior of a satellite lateral behavior in structure design. 15th International Mechanic Conference, Amirkabir University of Technology, in Persian. 
Safarabadi, M., \& Emami, H. (2009). Model updating of a micro satellite structure based on random vibration test result. $7^{\text {th }}$ International Aerospace Conference, Sahrif university of Technology, in Persian.

Sarafin, T. P., \& Larson, W. J. (1995). Spacecraft structures and mechanisms: from concept to launch. Wijker, J. J. (2003). Mechanical vibrations in spacecraft design. Springer Science \& Business Media. 